\title{
Leveraging Surface Siloxide Electronics to Enhance Relaxation Properties of a Single-Molecule Magnet
}

\author{
Maciej D. Korzyński ${ }^{\mathrm{a}}$, Zachariah J. Berkson ${ }^{\mathrm{a}}$, Boris Le Guennic ${ }^{*, b}$, \\ Olivier Cador*,b, and Christophe Copéret*,a
}

a Department of Chemistry and Applied Biosciences, ETH Zürich, Vladimir-Prelog Weg 1-5/10, 8093 Zürich, Switzerland

bUniv Rennes, CNRS, ISCR (Institut des Sciences Chimiques de Rennes), UMR 6226, 35000 Rennes, France

*boris.leguennic@univ-rennes1.fr, olivier.cador@univ-rennes1.fr, ccoperet@ethz.ch

\begin{abstract}
Single-molecule magnets (SMMs) hold promise for unmatched information storage density as well as applications in quantum computing and spintronics. To date, the most successful SMMs are organometallic lanthanide complexes. However, their surface immobilization, one of the requirements for device fabrication and commercial application, remains challenging due to sensitivity of magnetic properties to small changes in the electronic structure of the parent SMM. Thus, finding controlled approaches to SMM surface deposition is a timely challenge. In this contribution we apply the concept of isolobality to identify siloxides present at the surface of partially dehydroxylated silica as a suitable replacement for archetypal ligand architectures in organometallic SMMs. We demonstrate theoretically and experimentally that isolated siloxide anchorages not only enable successful immobilization, but also lead to two-orders-of-magnitude increase in magnetization relaxation times.
\end{abstract}




\section{Introduction}

The first disclosure[1] of a molecule exhibiting slow relaxation of magnetization in the absence of an external magnetic field has revolutionized magnetochemistry and opened a prolific field of research in molecular inorganic chemistry. Such single-molecule magnets (SMMs) could enable dramatic increase of information storage density that is not feasible with current technologies and they are thus attractive for quantum computing and spintronic applications. [2-7] State-of-the-art SMMs are assembled from lanthanide ions, which are associated with high intrinsic magnetic moments.[8-10] This property alone is not sufficient for slow relaxation of magnetization or the associated magnetic remanence. It is the presence of magnetic anisotropy, a preferential orientation of the magnetic moment in the molecule, that differentiates a SMM from a classical paramagnetic complex. This anisotropy is engendered by the crystal field induced by the ligands surrounding the metal center, and thus precise control over the metal coordination environment is of utmost importance in rational design of molecules exhibiting SMM behavior. For example, in the case of oblate[11] ions such as Dy(III) a highly axial crystal field is desired as exemplified by the remarkably high barrier for magnetic relaxation predicted for the hypothetical linear $[\mathrm{Dy}-\mathrm{O}]^{+}$fragment.[12] For experimentally realized systems, the best-performing SMMs to date are bespoke organometallic complexes based on a single Dy(III) center ligated with substituted cyclopentadienyl $\left(\mathrm{Cp}^{-}\right)$moieties.[13-16]

However, the industrial and commercial implementation of SMMs relies not only on the improvement of their intrinsic magnetic properties, but also their incorporation into solid-state devices.[17] Compared with the landmark developments in design, synthesis and understanding of SMMs, their deposition on material surfaces has received much less attention in the literature, likely because it still constitutes a significant challenge.[18] Indeed, efficient surface immobilization of SMMs requires the preservation of the magnetic performance of the parent precursor, while even minute alterations in the coordination sphere, including surface interactions, can lead to change or even a loss of SMM properties.[19] Hence, it is not surprising that immobilization strategies of SMMs on surfaces (Figure 1a, left) have mostly focused on minimizing changes in the first coordination sphere of the magnetic center upon deposition. Even with compelling methodologies such as formation of self-assembled monolayers, grafting on surfaces prefunctionalized with moieties present in the parent magnetic molecules, and simple adsorption onto the surface or within the pores of the solid matrix, the improvement or even preservation of the parent SMM's magnetic behavior is still rare, with some notable exceptions.[20-31] In contrast to the 'minimal perturbation' concept described above some groups explored deposition of single lanthanide atoms on the surfaces, [32-35] where direct interaction of the support with the metal center induces magnetic anisotropy (Figure 1a, right).[36] 


\section{a)}

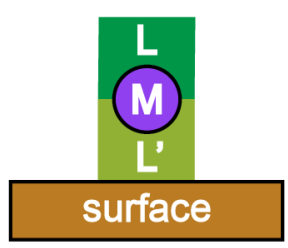

'Minimal perturbation' approach

b)

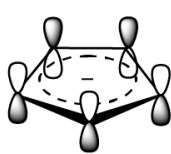

Tा

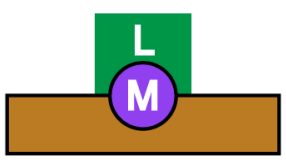

This work

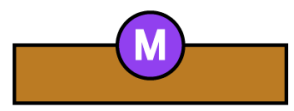

Single

atom deposition

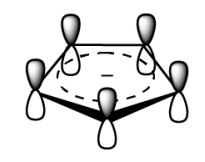

$\sigma$

c)

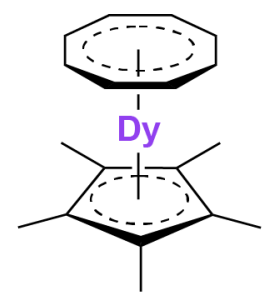

parent SMM
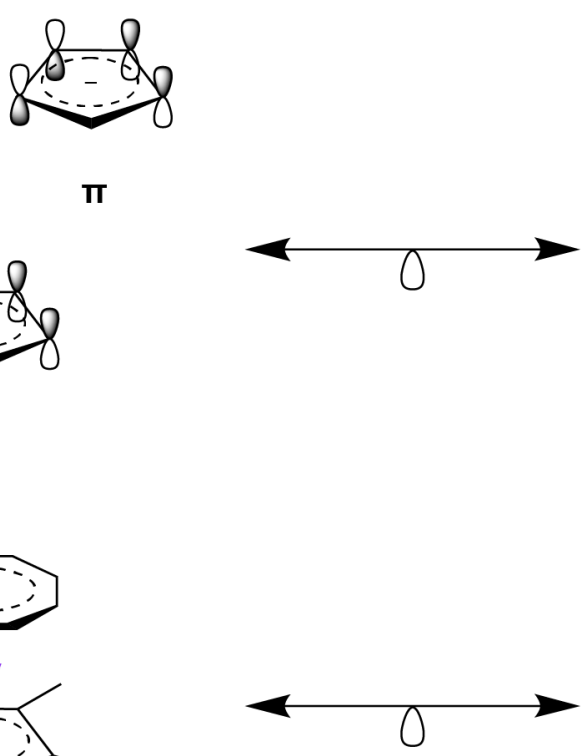

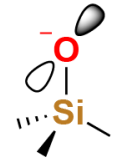<smiles>C[Si](C)(C)O[Na]</smiles>

$\pi$<smiles>C[Si](C)(C)O[O]</smiles>

$\sigma$

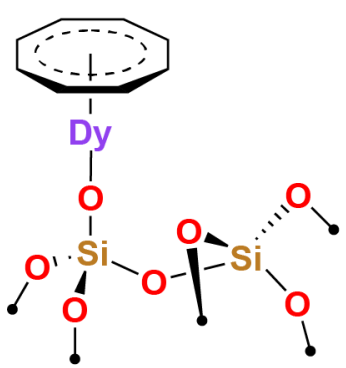

SMM on $\mathrm{SiO}_{2}$

Figure 1: (a) Schematic representation of state-of-the-art approaches to surface immobilization of SMMs along with the proposed methodology. (b) The analogy between the frontier orbitals of $\mathrm{Cp}^{-}$and siloxide moieties. (c) Same analogy shown for the SMM used in the study and the surface of $\mathrm{SiO}_{2}$. 
The lanthanide sites isolated in such fashion are the exemplars of the SMM concept, offering highest theoretical information density with single atom as a storage medium. Although very promising, this approach suffers from the challenges associated with vacuum deposition techniques such as high cost and scale-up difficulties. [37] In addition, the resulting coordination environment of the metal center is difficult to precisely control.

We decided to bridge the gap between the 'minimal perturbation' approach and single atom deposition on surfaces by SMM heterogenization using the surface organometallic chemistry (SOMC) methodology (Figure 1a, center).[38, 39] At its core, the SOMC approach involves controlled grafting of tailored metal fragments by protonolysis of a reactive anionic ligand of a molecular precursor on spatially separated $\mathrm{OH}$ groups at the surface of oxide supports. We reasoned that an isolated surface siloxy group, present at the surface of partially dehydroxylated silica, would be an ideal platform for this study as it readily offers the advantage of magnetic site dilution at the surface (minimizing magnetic dipolar interactions between adjacent metal centers). Furthermore, from frontier molecular orbital considerations, $\equiv \mathrm{SiO}^{-}$and $\mathrm{Cp}^{-}$act both as $6 \mathrm{e}^{-}$donor ligands; [40] they are formally isolobal (Figure 1b). Thus, in the first approximation, one could expect a siloxide moiety to act as a mimic for a cyclopentadienide ring, a ligand successfully used in the field of molecular magnetism. [41] Finally, the $\equiv \mathrm{SiO}^{-}$functionality can be viewed as an almost ideal negative point charge and thus should be capable of engendering much stronger axial crystal fields than $\mathrm{Cp}^{-}$, where the displacement of the lone pairs located on carbon nuclei from the main axis of the aromatic ring has to be considered.[42, 43] In turn, this should bolster the SMM properties of oblate lanthanide ions such as Dy(III). Herein, we evaluate this hypothesis first by theoretical calculations followed by grafting of an established organometallic SMM, (COT)Dy $\left(\mathrm{Cp}^{*}\right),[44,45]$ a neutral complex that contains one pentamethylcyclopentadienide $\left(\left(\mathrm{Cp}^{*}\right)^{-}\right)$moiety available for replacement with a surface siloxide group (Figure 1c). Owing to the charge and size of the cyclooctatetraenide $\left(\mathrm{COT}^{2-}\right)$ ligand one could anticipate formation of surface-bound and almost linear $\equiv \mathrm{SiO}-\mathrm{Dy}-\mathrm{COT}$ species. In this scenario, the SMM properties of $\mathrm{Dy}(\mathrm{III})$ ions should be enhanced by strong interactions with the surface negative charges, while maintaining a similar coordination environment to the parent SMM. In parallel, we explore the same approach with the yttrium(III) derivative acting as a diamagnetic chemical surrogate[46] for the Dy(III) species adding a powerful spectroscopic handle in the form of multinuclear nuclear magnetic resonance (NMR) spectroscopy. 

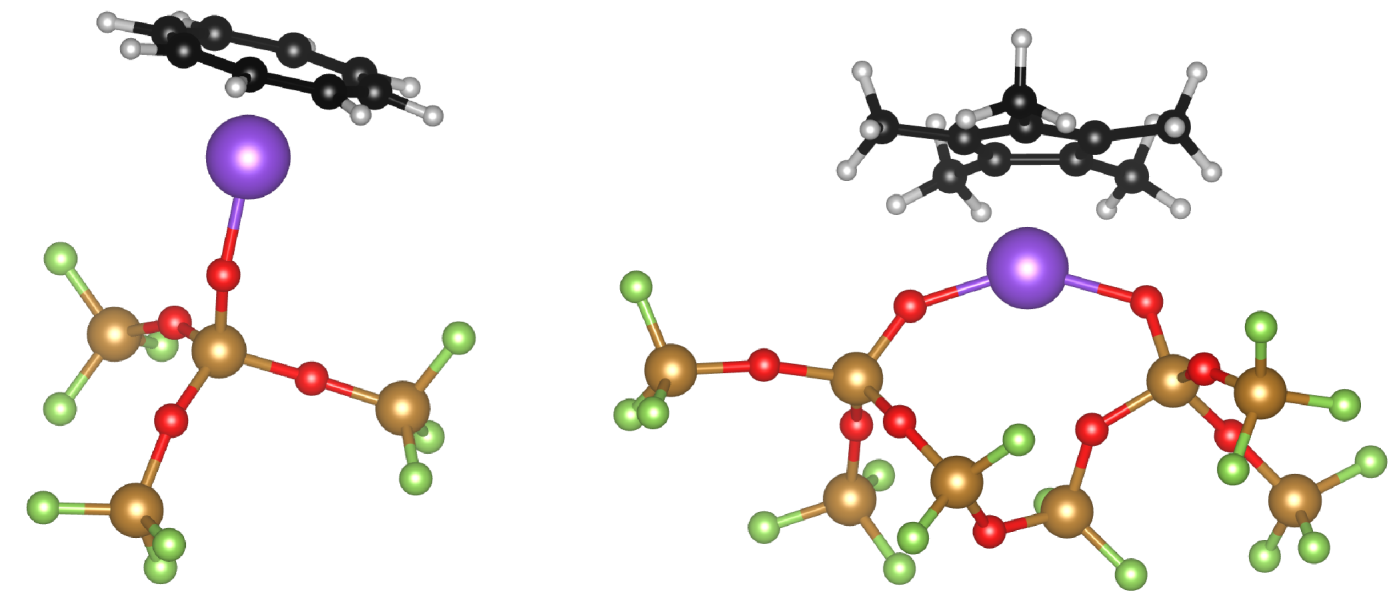

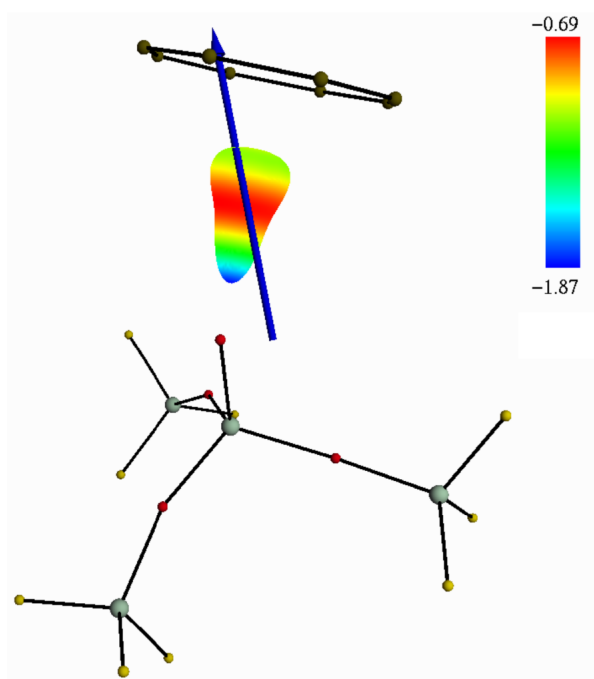

M1

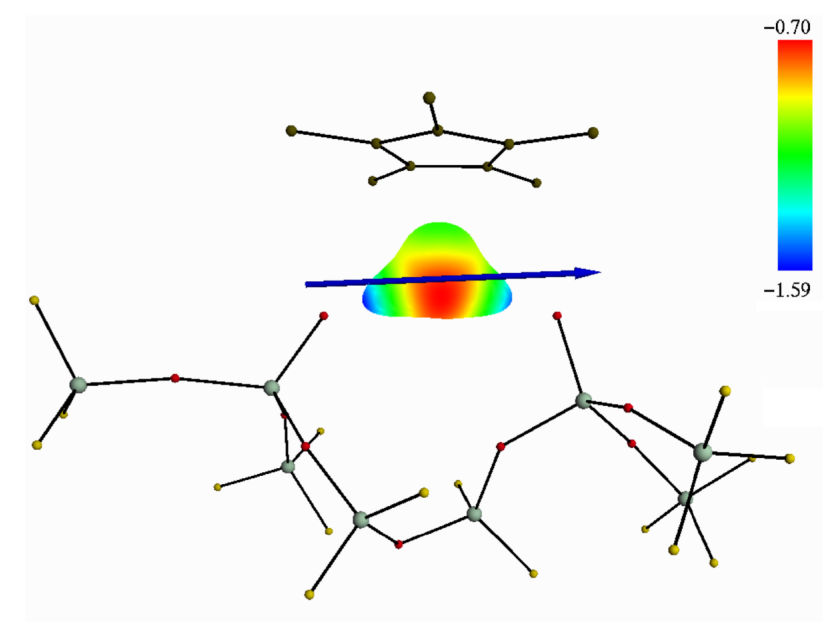

M4

Figure 2: Representative model of $\equiv \mathrm{SiO}-\mathrm{M}-\mathrm{COT}(\mathbf{M} 1)$ and $(\equiv \mathrm{SiO})_{2}-\mathrm{M}-\mathrm{Cp}^{*}(\mathbf{M} 4)$ species used in prediction of magnetic properties (top) and their calculated total electrostatic potentials (expressed in $\mathrm{e}^{-} \cdot \mathrm{bohr}^{-1}$ ) at $2.5 \AA$ around the Dy(III) ion with $\mathrm{g}_{\mathrm{z}}$ direction in blue (bottom). Additional models are depicted in the Supporting Information file. 


\section{Results and discussion}

We hypothesized that substitution of $\left(\mathrm{Cp}^{*}\right)^{-}$ligand for a surface siloxide will beneficially influence the magnetic performance compared to the parent magnetic precursor. This hypothesis was assessed computationally using theoretical models of the relevant species. Using the reported[47] periodic model of amorphous silica surface containing $1.1 \mathrm{OH}$ groups per $\mathrm{nm}^{2}$ as a starting point, several $\equiv \mathrm{SiO}-\mathrm{M}-\mathrm{COT}$ structures were optimized varying the geometry around the metal center and proximity of the ring to the surface itself (Figure S1, M1-M3). In addition to the $\equiv \mathrm{SiO}-\mathrm{M}-\mathrm{COT}$ species, we also envisioned the possibility of the formation of $(\equiv \mathrm{SiO})_{2}-\mathrm{M}-\mathrm{Cp}^{*}$ moieties via protonation of the formally di-anionic $\mathrm{COT}^{2-}$ ligand during grafting on silanols (Figure S1, M4). Cluster models were extracted from these periodic structures (M1-M4, Figures 2 and S2) and used as an input for CASSCF/SI-SO calculations (details in the Supporting Information file) along with the published crystal structure of (COT)Dy $\left(\mathrm{Cp}^{*}\right)$ for benchmarking. Comparison of the computed ordering for the Kramers doublets for all of the hypothetical $\equiv \mathrm{SiO}-\mathrm{Dy}-\mathrm{COT}$ structures shows expected variability in energy levels associated with slight changes in the geometry around metal center (Table S1). The variation of the energy levels notwithstanding, the striking feature of all calculated models is much larger energy differences between the ground and excited states when compared to the parent molecular SMM, (COT)Dy $\left(\mathrm{Cp}^{*}\right)$ (Table S1). Additionally, in hypothetical M2 and M3 structures the ground state Kramers doublet is almost pure $M_{J}= \pm 15 / 2$. Similar analysis of the $(\equiv \mathrm{SiO})_{2}-\mathrm{M}-\mathrm{Cp}^{*}$ model $(\mathrm{M} 4)$ also reveals a ground state - excited state gap larger than for the molecular precursor (Table S1). In this case the orientation of the primary component of the g-tensor (Figure S3) is much different than in models M1M3, yet still the ground state wavefunction has primarily $\mathrm{M}_{\mathrm{J}}= \pm 15 / 2$ contributions. In M1-M3, the main component of g-tensor is almost parallel to Dy-O bond and thus its orientation is mainly driven by negative electrostatic potential associated with the surface siloxide oxygen atom (Figures 2 and S4).[48, 49] In M4, the presence of two bound oxygen atoms induces an orientation of the magnetic axis parallel to the surface. Moreover, transition magnetic moment probabilities for $\mathbf{M} 1-\mathbf{M} 4$ reveal that the relaxation process involves excited states (thermally activated mechanism) without quantum tunnelling of magnetization (QTM) between the ground state Kramers doublets (Figure S5). Calculations performed for M1-M3 validate the hypothesis that a $\equiv \mathrm{SiO}^{-}$moiety, while formally isolobal to $\left(\mathrm{Cp}^{*}\right)^{-}$, creates a stronger crystal field leading to potentially better SMM. This is further supported by computational results on $\mathbf{M} 4$, where two $\equiv \mathrm{SiO}^{-}$moieties rather than $\left(\mathrm{Cp}^{*}\right)^{-}$drive the magnetic properties. With those theoretical predictions in hand, we explored surface chemistry and magnetism of the (COT)M(Cp*) system.

Treatment of partially dehydroxylated silica $\left(\mathrm{SiO}_{2}\right.$ with $0.37 \mathrm{mmol}$ of accessible silanols 
per gram) with toluene solutions of either $(\mathrm{COT}) \mathrm{Y}\left(\mathrm{Cp}^{*}\right)(\mathbf{1})$ or $(\mathrm{COT}) \operatorname{Dy}\left(\mathrm{Cp}^{*}\right)(\mathbf{2}, 1$ equiv. with respect to silanol groups) at $-20{ }^{\circ} \mathrm{C}$ leads to an instantaneous change of the silica's color from white to dark red. After three-hour periods, during which the mixtures were allowed to reach room temperature, the supernatant was removed and the material was washed with fresh toluene. The isolated materials were dried for 12 hours under high-vacuum $\left(10^{-5}\right.$ mbar $)$ to yield free-flowing magenta powders denoted $\mathbf{1} / \mathrm{SiO}_{\mathbf{2}}$ and $\mathbf{2} / \mathrm{SiO}_{2}$. Transmission FTIR spectra (Figure 3a) of silica before and after grafting indicate almost complete consumption of the isolated silanol groups $\left(3747 \mathrm{~cm}^{-1}\right)$ and emergence of C-H stretching modes (3100-2800 $\left.\mathrm{cm}^{-1}\right)$ as well as the feature characteristic for $\mathrm{COT}^{2-}\left(712 \mathrm{~cm}^{-1}\right)$, which can also be observed in the FTIR spectra of both precursors (Figure S7). A solution ${ }^{1} \mathrm{H}$ NMR spectrum obtained from an analogous grafting experiment performed in benzene- $d 6$ revealed pentamethylcyclopentadiene $\left(\mathrm{Cp}^{*} \mathrm{H}\right)$ and a mixture of trienes (cycloocta-1,3,5-triene and cycloocta-1,3,6-triene) as sole organic products in the reaction supernatant, identified based on comparison with protonation studies performed on $\mathbf{1}$ (Figure S17 and further discussion in the Supporting Information file). Additionally, the solution ${ }^{1} \mathrm{H}$ NMR spectra acquired during formation of $1 / \mathrm{SiO}_{2}$ and $2 / \mathrm{SiO}_{2}$ are nearly identical, indicating that the process occurs in a qualitatively similar manner for both molecular precursors (Figure S18).

Formation of $\mathrm{Cp}^{*} \mathrm{H}$ and trienes during grafting suggests that both ligands present in the molecular precursors can undergo protonation by surface silanol moieties, which implies the presence of multiple types of metal-containing surface species. To directly determine the types of surface metal environments resulting from grafting of $(\mathrm{COT}) \mathrm{M}\left(\mathrm{Cp}^{*}\right)$ species on silica, we analyzed the $1 \mathrm{D}$ and $2 \mathrm{D}{ }^{1} \mathrm{H}$ and ${ }^{13} \mathrm{C}$ solid-state magic angle spinning (MAS) NMR spectra of $1 / \mathrm{SiO}_{2}$. The solid-state ${ }^{1} \mathrm{H}$ MAS echo spectrum of $\mathbf{1} / \mathrm{SiO}_{\mathbf{2}}$ (Figure 3b, top), shows ${ }^{1} \mathrm{H}$ signals at 6.3 and $5.7 \mathrm{ppm}$ from ${ }^{1} \mathrm{H}$ species on $\mathrm{COT}^{2-}$ bound to $\mathrm{Y}$ or adsorbed trienes and an inhomogeneously broadened feature at $1.7 \mathrm{ppm}$ that could arise from $\left(\mathrm{Cp}^{*}\right)^{-}$bound to $\mathrm{Y}$ or adsorbed $\mathrm{Cp} * \mathrm{H}$. Though the $1 \mathrm{D}{ }^{1} \mathrm{H}$ MAS NMR spectrum is relatively poorly resolved, the $2 \mathrm{D}{ }^{1} \mathrm{H}\left\{{ }^{13} \mathrm{C}\right\}$ dipolar-mediated heteronuclear multiquantum coherence (D-HMQC) spectrum of $1 / \mathrm{SiO}_{2}$ in Figure $3 \mathrm{~b}$ reveals narrow and well-resolved correlated ${ }^{13} \mathrm{C}-{ }^{1} \mathrm{H}$ signals arising from surface-bound $\equiv \mathrm{SiO}-\mathrm{Y}-\mathrm{COT}$ and $(\equiv \mathrm{SiO})_{2}-\mathrm{Y}-\mathrm{Cp} *$ species. Specifically, the ${ }^{1} \mathrm{H}$ signals at 6.3 and $5.7 \mathrm{ppm}$ are both correlated to a ${ }^{13} \mathrm{C}$ signal at $94 \mathrm{ppm}$, which is assigned to surface $\mathrm{COT}^{2-}$ bound to $\mathrm{Y}$ on the basis of ${ }^{13} \mathrm{C}$ NMR analyses of the molecular precursor before and after solution protonation experiments (further details in Supporting Information). No ${ }^{13} \mathrm{C}$ signals associated with adsorbed trienes (expected in the $140-125 \mathrm{ppm}$ range and at $\approx 28 \mathrm{ppm}$ ) are detected, indicating that such species are not present within the detection limits of the measurement. Thus, the ${ }^{1} \mathrm{H}$ signals at 6.3 and $5.7 \mathrm{ppm}$ arise from different types of 


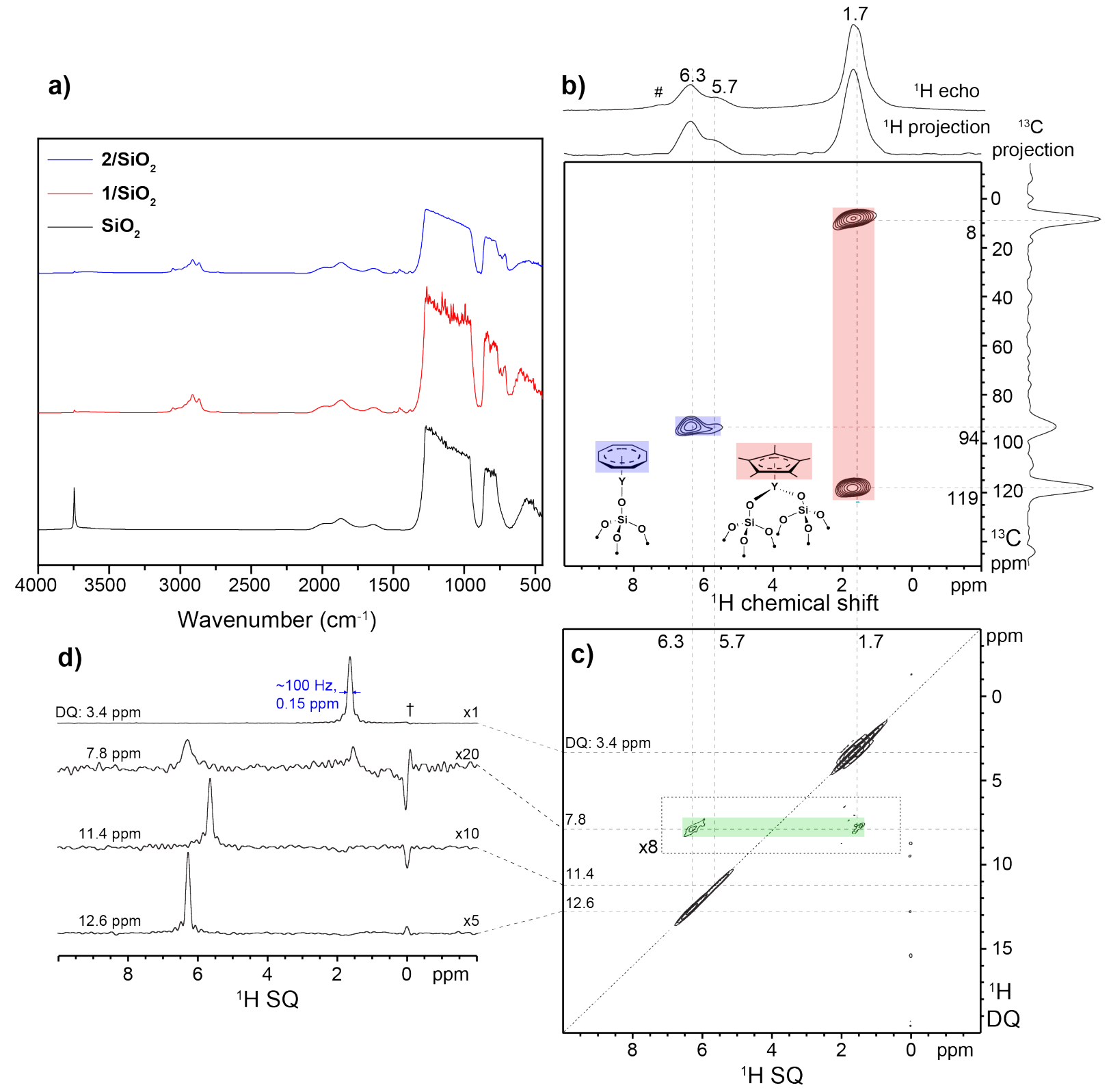

Figure 3: (a) Comparison of transmission FTIR spectra of $\mathrm{SiO}_{2}$ before (black) and after grafting of $\mathbf{1}$ (red) or $\mathbf{2}$ (blue). Solid state (b) $2 \mathrm{D}{ }^{1} \mathrm{H}\left\{{ }^{13} \mathrm{C}\right\}$ D-HMQC and (c) 2D ${ }^{1} \mathrm{H}\left\{{ }^{1} \mathrm{H}\right\}$ SQ-DQ NMR correlation spectra of $\mathbf{1} / \mathrm{SiO}_{2}$ acquired at $16.4 \mathrm{~T}, 50 \mathrm{kHz} \mathrm{MAS}$, and ambient temperature. A solid-state $1 \mathrm{D}^{1} \mathrm{H}$ echo MAS NMR spectrum acquired under the same conditions is shown along the horizontal axis in (b) for comparison with the $2 \mathrm{D}$ spectra. The \# symbol indicates a ${ }^{1} \mathrm{H}$ background signal. (d) Horizontal SQ slices extracted from the 2D spectrum in (c) at the indicated DQ shift positions. The $†$ symbol indicates $t_{1}$ artifacts. 
surface $\equiv \mathrm{SiO}-\mathrm{Y}-\mathrm{COT}$ species, likely having different local environments. A solid-state ${ }^{89} \mathrm{Y}\left\{{ }^{1} \mathrm{H}\right\}$ NMR spectrum of $\mathbf{1} / \mathrm{SiO}_{\mathbf{2}}$ also indicates the presence of such species (Figure $\mathrm{S} 21$, details in the Supporting Information file). The ${ }^{1} \mathrm{H}$ signal at $1.7 \mathrm{ppm}$ (Figure $3 \mathrm{~b}$ ) is correlated to ${ }^{13} \mathrm{C}$ signals at 8 and $119 \mathrm{ppm}$, which are assigned respectively to the methyl and ring carbons of $(\equiv \mathrm{SiO})_{2}-\mathrm{Y}-\mathrm{Cp}^{*}$ species. Analysis of the weight percentages of $\mathrm{C}$ and $\mathrm{H}$ indicate the possible presence of additional organics such as adsorbed $\mathrm{Cp} * \mathrm{H}$. However, ${ }^{1} \mathrm{H}$ signals from surface-bound $\mathrm{Cp} * \mathrm{H}$ and $(\equiv \mathrm{SiO})_{2}-\mathrm{Y}-\mathrm{Cp}^{*}$ likely overlap in the solid-state NMR spectra and the ${ }^{13} \mathrm{C}$ signals from dilute surface $\mathrm{Cp} * \mathrm{H}$ may be too weak to detect. Overall, the $2 \mathrm{D}{ }^{1} \mathrm{H}\left\{{ }^{13} \mathrm{C}\right\}$ HMQC spectrum directly establishes the mutual coexistence of distinct surface $\equiv \mathrm{SiO}-\mathrm{Y}-\mathrm{COT}$ and $(\equiv \mathrm{SiO})_{2}-\mathrm{Y}-\mathrm{Cp}{ }^{*}$ species in $\mathbf{1} / \mathrm{SiO}_{\mathbf{2}}$. Analyses of ${ }^{1} \mathrm{H} T_{2}$ relaxation times (details in the Supporting Information) indicate that the organic moieties bound to Y centers maintain a high degree of molecular mobility as expected from their "free-standing" character and that the surface-bound COT and Cp* species are present in an approximate 1:1 ratio.

The surface organometallic species in $1 / \mathrm{SiO}_{2}$ exhibit extremely well-defined local environments and are predominantly well-dispersed and non-interacting. This is established by analysis of the $2 \mathrm{D}{ }^{1} \mathrm{H}\left\{{ }^{1} \mathrm{H}\right\}$ single-quantum double-quantum (SQ-DQ) NMR correlation spectrum in Figure 3c. The 2D SQ-DQ correlation spectrum yields remarkably narrow $(\approx 0.15$ ppm full-width-half-maximum, FWHM) linewidths for the on-diagonal signals at SQ: $1.7,5.7$, and $6.3 \mathrm{ppm}$, as shown in the horizontal slices extracted from the $2 \mathrm{D}$ spectrum at different DQ shift positions in Figure $3 \mathrm{~d}$. The relatively broad ${ }^{1} \mathrm{H}$ signals (0.5 to $1.0 \mathrm{ppm}$ FWHM) at these positions in the $1 \mathrm{D}{ }^{1} \mathrm{H}$ MAS NMR spectra thus manifest the superposition of very narrow overlapping signals arising from distributions of locally ordered surface $\mathrm{COT}^{2-}$ and $\left(\mathrm{Cp}^{*}\right)^{-}$moieties. Only a small subset of the $\mathrm{COT}^{2-}$ and $\left(\mathrm{Cp}^{*}\right)^{-}$species are close enough to interact, as manifested by the correlated off-diagonal signals at SQ: 1.7 and $6.3 \mathrm{ppm}$ (green shaded region in Figure 3c). This signal intensity pair arises from $\mathrm{COT}^{2-}$ and $\left(\mathrm{Cp}^{*}\right)^{-}$species that are dipole-dipole coupled over nanoscale distances $(<0.5 \mathrm{~nm})$. This can be due to $\equiv \mathrm{SiO}-\mathrm{Y}-\mathrm{COT}$ interacting with nearby $(\equiv \mathrm{SiO})_{2}-\mathrm{Y}-\mathrm{Cp} *$ or $\equiv \mathrm{SiO}-\mathrm{Y}-\mathrm{COT}$ interacting with adsorbed $\mathrm{Cp}^{*} \mathrm{H}$. The latter case seems most plausible as the protonolysis of $\mathbf{1}$ at a single $\equiv \mathrm{SiOH}$ site releases $\mathrm{Cp} * \mathrm{H}$, which could adsorb at a nearby silanol and interact with the surface $\equiv \mathrm{SiO}-\mathrm{Y}-\mathrm{COT}$. To assess the interaction of $\mathrm{Cp} * \mathrm{H}$ on the surface of silica, we contacted $\mathrm{SiO}_{2}$ with a solution of $\mathrm{Cp}^{*} \mathrm{H}$ in toluene followed by washing with solvent and drying under high vacuum. Subsequent ${ }^{1} \mathrm{H}$ MAS NMR measurements revealed ${ }^{1} \mathrm{H}$ signal at $1.7 \mathrm{ppm}$ from adsorbed $\mathrm{Cp}^{*} \mathrm{H}$ (Figure S24) proving the interaction of this olefin with surface is strong enough to withstand post-reaction treatment. To sum up, the $2 \mathrm{D}{ }^{1} \mathrm{H}\left\{{ }^{1} \mathrm{H}\right\}$ correlation spectrum reveals that the surface $\equiv \mathrm{SiO}-\mathrm{Y}-\mathrm{COT}$ and $(\equiv \mathrm{SiO})_{2}-\mathrm{Y}-\mathrm{Cp}^{*}$ moieties exhibit a surprising 


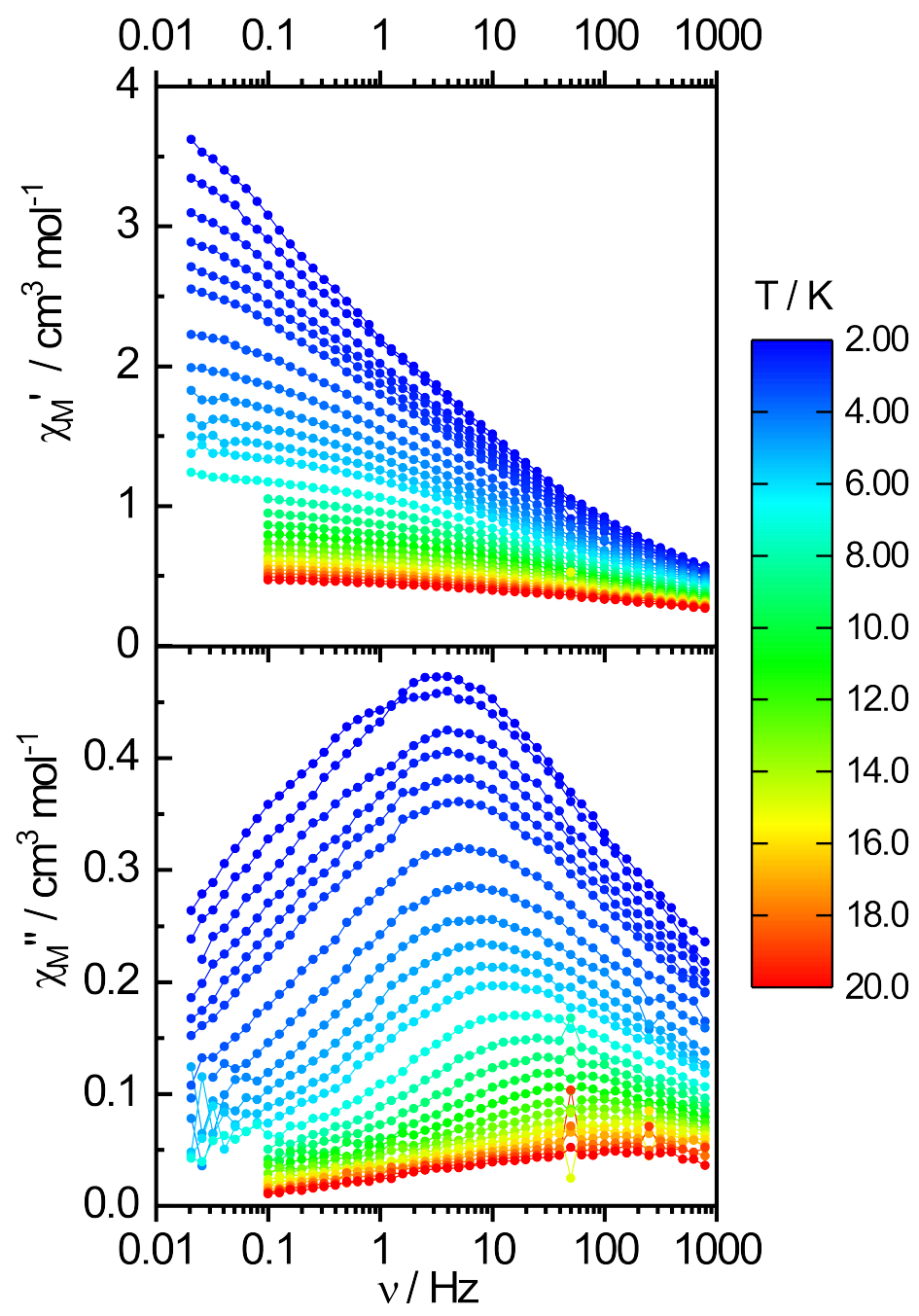

Figure 4: Frequency dependence of both in-phase (top) and out-of-phase (down) components of the AC susceptibility measured in zero external DC field between 2 and $20 \mathrm{~K}$ for $2 / \mathrm{SiO}_{2}$. Circles represent experimental data points; the lines do not have any physical meaning.

degree of local order despite the complexity of the surface and the grafting mixture.

$\mathrm{AC}$ magnetic measurements on $\mathbf{2} / \mathrm{SiO}_{2}$ show slow relaxation of the magnetization in the absence of the external DC field (Figure 4, see Supporting Information for experimental details). Most notably, the zero-field relaxation time of $\mathbf{2} / \mathbf{S i O}_{\mathbf{2}}$ at $2 \mathrm{~K}$ is approximately two orders of magnitude slower than that of the magnetically diluted molecular precursor (COT)Dy $\left(\mathrm{Cp}^{*}\right),[44]$ confirming our theoretical predictions regarding the beneficial influence of the siloxide group on magnetic properties of the oblate Dy(III) center. To the best of our knowledge this represents the largest increase in remanence time upon surface immobilization compared to diluted parent SMM reported so far. The out-of-phase component of the magnetic susceptibility, $\chi_{M}$, passes through a maximum at oscillation frequencies of the $\mathrm{AC}$ driving field that vary with temperature 
and can be fitted with extended Debye model between 2 and $17 \mathrm{~K}$ (Table S5 and Figure S27). The thermal variation of the relaxation time (Figure S28) of the magnetic moment reveals that the Raman relaxation process $\left(\tau^{-1}=C T^{n}\right)$ is predominant while QTM and Orbach (over the barrier) processes are drastically slower. Quantitatively, a very good agreement is obtained with $\mathrm{C}=1.847 \mathrm{~s}^{-1} \cdot \mathrm{K}^{-\mathrm{n}}$ and $\mathrm{n}=2.246$. While such relaxation characteristics preclude extraction of the effective barrier to magnetization reversal, and thus direct correlation of the experimental data with our models, the observed behavior aligns well with the conclusions of our theoretical predictions, where negligible QTM is expected from the $\equiv \mathrm{SiO}-\mathrm{M}-\mathrm{COT}$ models (M1-M3). Despite the high $\alpha$ value $(>0.5)$ that represents a large distribution of relaxation times there is no evidence of additional relaxation processes. Applying DC fields during AC measurements results in shifting of maximum in $\chi_{M}=f(\nu)$ curves to lower frequencies (Figure S29). At 1000 Oe the relaxation time is extended approximately by an additional order of magnitude compared with measurements performed in the absence of the static field. The room temperature value of $\chi_{M} T$ product was determined to be $10.5 \mathrm{~cm}^{3} \cdot \mathrm{K} \cdot \mathrm{mol}^{-1}$ (Figure S30); at low temperatures the value decreases precipitously in a fashion similar to the precursor 2. [44] The hysteresis loop at $2 \mathrm{~K}$ and $16 \mathrm{Oe} \cdot \mathrm{s}^{-1}$ (Figure S31) is open in field with butterfly shape and persistent coercivity in zero field $\left(\mathrm{H}_{\mathrm{C}}=200 \mathrm{Oe}\right)$. The calculated magnetization curves at $2 \mathrm{~K}$ (Figure S31) reproduce saturation magnetization $(\approx 4 \mathrm{~N} \beta)$ for all models and fall within the experimental hysteresis loop.

\section{Conclusion}

In this contribution we expanded the suite of SMM surface immobilization techniques. Using a theory-driven approach we surmised that siloxide moieties present on the surface of partially dehydroxylated silica are not only a suitable replacement for ligands supporting known organolanthanide SMMs from the electronic point of view, but can also lead to an improvement of magnetic properties. Using (COT)Dy $\left(\mathrm{Cp}^{*}\right)$ as a test system we demonstrated a possibility of magnetization relaxation time extension by two orders of magnitude on surface deposition compared to the parent SMM cocrystallized in a diamagnetic matrix. The methodology presented herein holds promise for broader applicability in the field of molecular magnetism, in particular for preeminent oblate ion-based organolanthanide systems.

\section{Conflict of interest}

There are no conflicts to declare. 


\section{Acknowledgements}

M.D.K. would like to thank ETH Zurich Postdoctoral Fellowships program (FEL-23 191). Z.J.B. acknowledges support from the Swiss National Science Foundation (SNSF) Spark program (grant no. CRSK-2_190322). B.L.G. thanks the French GENCI/IDRISCINES center for high-performance computing resources. Anton Ashuiev is acknowledged for collection of the low-temperature EPR data.

\section{References}

1. Sessoli, R., Gatteschi, D., Caneschi, A. \& Novak, M. A. Magnetic bistability in a metal-ion cluster. Nature 365, 141-143 (1993).

2. Liddle, S. T. \& van Slageren, J. Improving f-element single molecule magnets. Chem. Soc. Rev. 44, 6655-6669 (2015).

3. Santini, P., Carretta, S. \& Amoretti, G. in Molecular Magnetic Materials: Concepts and Applications (eds Sieklucka, B. \& Pinkowicz, D.) 103-129 (Wiley-VCH Verlag GmbH \& Co. KGaA, 2016).

4. Gaita-Ariño, A. et al. Coherence and organisation in lanthanoid complexes: From single ion magnets to spin qubits. Inorg. Chem. Front. 3, 568-577 (2016).

5. Komeda, T., Katoh, K. \& Yamashita, M. in Molecular Technology (eds Yamamoto, H. \& Kato, T.) 263-304 (Wiley-VCH Verlag GmbH \& Co. KGaA, 2019).

6. Gaita-Ariño, A., Luis, F., Hill, S. \& Coronado, E. Molecular spins for quantum computation. Nat. Chem. 11, 301-309 (2019).

7. Spree, L. \& Popov, A. A. Recent advances in single molecule magnetism of dysprosiummetallofullerenes. Dalton Trans. 48, 2861-2871 (2019).

8. Carlin, R. L. in Magnetochemistry 237-261 (Springer Berlin Heidelberg, 1986).

9. Luzon, J. \& Sessoli, R. Lanthanides in molecular magnetism: So fascinating, so challenging. Dalton Trans. 41, 13556-13567 (2012).

10. Woodruff, D. N., Winpenny, R. E. P. \& Layfield, R. A. Lanthanide Single-Molecule Magnets. Chem. Rev. 113, 5110-5148 (2013).

11. Rinehart, J. D. \& Long, J. R. Exploiting single-ion anisotropy in the design of f-element single-molecule magnets. Chem. Sci. 2, 2078-2085 (2011).

12. Ungur, L. \& Chibotaru, L. F. Magnetic anisotropy in the excited states of low symmetry lanthanide complexes. Phys. Chem. Chem. Phys. 13, 20086-20090 (2011). 
13. Goodwin, C. A. P., Ortu, F., Reta, D., Chilton, N. F. \& Mills, D. P. Molecular magnetic hysteresis at 60 kelvin in dysprosocenium. Nature 548, 439-442 (2017).

14. Guo, F.-S. et al. A Dysprosium Metallocene Single-Molecule Magnet Functioning at the Axial Limit. Angew. Chem. Int. Ed. 56, 11445-11449 (2017).

15. Guo, F.-S. et al. Magnetic hysteresis up to 80 kelvin in a dysprosium metallocene single-molecule magnet. Science 362, 1400-1403 (2018).

16. Randall McClain, K. et al. High-temperature magnetic blocking and magnetostructural correlations in a series of dysprosium(iii) metallocenium single-molecule magnets. Chem. Sci. 9, 8492-8503 (2018).

17. Harriman, K. L., Errulat, D. \& Murugesu, M. Magnetic Axiality: Design Principles from Molecules to Materials. Trends Chem. 1, 425-439 (2019).

18. Cornia, A., Mannini, M., Sainctavit, P. \& Sessoli, R. Chemical strategies and characterization tools for the organization of single molecule magnets on surfaces. Chem. Soc. Rev. 40, 3076-3091 (2011).

19. Holmberg, R. J. \& Murugesu, M. Adhering magnetic molecules to surfaces. J. Mater. Chem. C 3, 11986-11998 (2015).

20. Ruiz-Molina, D. et al. Isolated Single-Molecule Magnets on the Surface of a Polymeric Thin Film. Adv. Mater. 15, 42-45 (2003).

21. Kyatskaya, S. et al. Anchoring of Rare-Earth-Based Single-Molecule Magnets on Single-Walled Carbon Nanotubes. J. Am. Chem. Soc. 131, 15143-15151 (2009).

22. Mannini, M. et al. Magnetic memory of a single-molecule quantum magnet wired to a gold surface. Nat. Mater. 8, 194-197 (2009).

23. Giusti, A. et al. Magnetic Bistability of Individual Single-Molecule Magnets Grafted on Single-Wall Carbon Nanotubes. Angew. Chem. Int. Ed. 48, 4949-4952 (2009).

24. Mannini, M. et al. Quantum tunnelling of the magnetization in a monolayer of oriented single-molecule magnets. Nature 468, 417-421 (2010).

25. Gonidec, M. et al. Surface Supramolecular Organization of a Terbium(III) DoubleDecker Complex on Graphite and its Single Molecule Magnet Behavior. J. Am. Chem. Soc. 133, 6603-6612 (2011).

26. Kahle, S. et al. The Quantum Magnetism of Individual Manganese-12-Acetate Molecular Magnets Anchored at Surfaces. Nano Lett. 12, 518-521 (2012).

27. Malavolti, L. et al. Magnetic Bistability in a Submonolayer of Sublimated $\mathrm{Fe}_{4}$ SingleMolecule Magnets. Nano Lett. 15, 535-541 (2015). 
28. Kiefl, E. et al. Robust Magnetic Properties of a Sublimable Single-Molecule Magnet. ACS Nano 10, 5663-5669 (2016).

29. Wäckerlin, C. et al. Giant Hysteresis of Single-Molecule Magnets Adsorbed on a Nonmagnetic Insulator. Adv. Mater. 28, 5195-5199 (2016).

30. Nakanishi, R. et al. DySc ${ }_{2} \mathrm{~N} @ \mathrm{C}_{80}$ Single-Molecule Magnetic Metallofullerene Encapsulated in a Single-Walled Carbon Nanotube. J. Am. Chem. Soc. 140, 10955-10959 (2018).

31. Krylov, D. S. et al. Substrate-Independent Magnetic Bistability in Monolayers of the Single-Molecule Magnet $\mathrm{Dy}_{2} \mathrm{ScN} @ \mathrm{C}_{80}$ on Metals and Insulators. Angew. Chem. Int. Ed. 59, 5756-5764 (2020).

32. Donati, F. et al. Magnetic remanence in single atoms. Science 352, 318-321 (2016).

33. Baltic, R. et al. Superlattice of Single Atom Magnets on Graphene. Nano Lett. 16, 7610-7615 (2016).

34. Natterer, F. D. et al. Reading and writing single-atom magnets. Nature 543, 226228 (2017).

35. Allouche, F. et al. Magnetic Memory from Site Isolated Dy(III) on Silica Materials. ACS Cent. Sci. 3, 244-249 (2017).

36. Miyamachi, T. et al. Stabilizing the magnetic moment of single holmium atoms by symmetry. Nature 503, 242-246 (2013).

37. Chen, Y., Huang, Z., Ma, Z., Chen, J. \& Tang, X. Fabrication, characterization, and stability of supported single-atom catalysts. Catal. Sci. Technol. 7, 4250-4258 (2017).

38. Basset, J. M. \& Ugo, R. in Modern Surface Organometallic Chemistry 1-21 (John Wiley \& Sons, Ltd, 2009).

39. Copéret, C. et al. Surface Organometallic and Coordination Chemistry toward Single-Site Heterogeneous Catalysts: Strategies, Methods, Structures, and Activities. Chem. Rev. 116, 323-421 (2016).

40. Wolczanski, P. T. Chemistry of electrophilic metal centres coordinated by silox $\left({ }^{t} \mathrm{Bu}_{3} \mathrm{SiO}\right)$, tritox $\left({ }^{\mathrm{t}} \mathrm{Bu}_{3} \mathrm{CO}\right)$ and related binfunctional ligands. Polyhedron 14, 33353362 (1995).

41. Layfield, R. A. Organometallic Single-Molecule Magnets. Organometallics 33, 10841099 (2014). 
42. Baldoví, J. J., Borrás-Almenar, J. J., Clemente-Juan, J. M., Coronado, E. \& GaitaAriño, A. Modeling the properties of lanthanoid single-ion magnets using an effective point-charge approach. Dalton Trans. 41, 13705-13710 (2012).

43. Jiang, S.-D. \& Qin, S.-X. Prediction of the quantized axis of rare-earth ions: the electrostatic model with displaced point charges. Inorg. Chem. Front. 2, 613-619 (2015).

44. Jiang, S.-D. et al. Series of Lanthanide Organometallic Single-Ion Magnets. Inorg. Chem. 51, 3079-3087 (2012).

45. Harriman, K. L. M. \& Murugesu, M. An Organolanthanide Building Block Approach to Single-Molecule Magnets. Acc. Chem. Res. 49, 1158-1167 (2016).

46. Cotton, S. A. in Encyclopedia of Inorganic and Bioinorganic Chemistry (ed Scott, R. A.) (American Cancer Society, 2011).

47. Comas-Vives, A. Amorphous $\mathrm{SiO}_{2}$ surface models: energetics of the dehydroxylation process, strain, ab initio atomistic thermodynamics and IR spectroscopic signatures. Phys. Chem. Chem. Phys. 18, 7475-7482 (10 2016).

48. Zhang, K. et al. Tuning the Magnetic Interactions in Dy(III $)_{4}$ Single-Molecule Magnets. Inorg. Chem. 57, 8550-8557 (2018).

49. Huang, G. et al. Magnetic Slow Relaxation in a Metal-Organic Framework Made of Chains of Ferromagnetically Coupled Single-Molecule Magnets. Chem. Eur. J. 24, 6983-6991 (2018). 